\title{
Caracterização eletromagnética do compósito de carbeto de silício e negro de fumo em matriz polimérica
}

\author{
Electromagnetic characterization of the \\ silicon carbide and carbon black composite \\ in a polymeric matrix
}

Nila Cecília de Faria Lopes Medeiros ${ }^{1,2}$, Leonardo Iusuti de Medeiros ${ }^{1,2}$, Ariane Aparecida Teixeira de Souza ${ }^{2}$, Guilherme Frederico Bernardo Lenz e Silva ${ }^{3}$, Alan Fernando Ney Boss ${ }^{3}$, Gisele Aparecida Amaral-Labat ${ }^{2}$, Maurício Ribeiro Baldan ${ }^{2}$.

\footnotetext{
${ }^{1}$ Universidade Estadual de Santa Cruz, DCET, Rodovia Jorge Amado, Km 16, CEP: 45.662-900, Ilhéus, BA, Brasil ${ }^{2}$ Instituto Nacional de Pesquisas Espaciais, PG-ETE, Av. dos Astronautas, 1.758, Jardim da Granja, CEP: 12.227-010, São José dos Campos, SP, Brasil

${ }^{3}$ Universidade de São Paulo, Escola Politécnica, Avenida Professor Mello Moraes, 2463, CEP: 05.508-030, Cidade Universitária, São Paulo, SP, Brasil

e-mail:ncflmedeiros@uesc.br, limedeiros@uesc.br, guilhermelenz@usp.br, ariane_apts@hotmail.com, gisele.amarallabat@gmail.com,mauricio.baldan@inpe.br, alan.boss@usp.br
}

\section{RESUMO}

Este trabalho apresenta as propriedades eletromagnéticas de um Material Absorvedor de Radiação Eletromagnética (MARE) feito a partir de Carbeto de Silício (SiC) e Negro de Fumo (NF) como aditivos. O compósito $\mathrm{SiC} / \mathrm{NF}$ foi produzido utilizando $70 \%(\mathrm{~m} / \mathrm{m})$ de matriz de silicone, e variando as concentrações do aditivo em $3 \%(\mathrm{~m} / \mathrm{m}), 2 \%(\mathrm{~m} / \mathrm{m})$ e $1 \%(\mathrm{~m} / \mathrm{m})$ de $\mathrm{NF}$ com $27 \%(\mathrm{~m} / \mathrm{m}), 28 \%(\mathrm{~m} / \mathrm{m})$ e $29 \%(\mathrm{~m} / \mathrm{m})$ de SiC, respectivamente. A estrutura química e a morfologia das partículas de SiC e NF foram analisadas através de espectroscopia Raman e Microscopia Eletrônica de Varredura (MEV). As propriedades dielétricas dos MAREs foram obtidas por meio do analisador de rede vetorial na faixa de frequência de 12,4 a 18,0 GHz. Os resultados de refletividade mostraram que o compósito $\mathrm{SiC} / \mathrm{NF}$ com $1 \%(\mathrm{~m} / \mathrm{m})$ de $\mathrm{NF}$ apresentou a melhor absorção de radiação eletromagnética, chegando a $-47,9 \mathrm{~dB}$ em 16,8 GHz com uma amostra de aproximadamente 5,5 mm de espessura.

Palavras-chave: MARE, banda $\mathrm{K}_{\mathrm{u}}$, refletividade, permissividade, material dielétrico.

\section{ABSTRACT}

This work presents the electromagnetic properties of a Radar Absorbing Material (RAM) made with Silicon Carbide ( $\mathrm{SiC}$ ) and Carbon Black (CB) as additives. Composites of silicone rubber and SiC/CB were produced using $70 \mathrm{wt} \%$ of silicone rubber as matrix and a variation of $3 \mathrm{wt} \%, 2 \mathrm{wt} \%$ and $1 \mathrm{wt} \%$ of $\mathrm{CB}$ with $27 \mathrm{wt} \%, 28 \mathrm{wt} \%$ and $29 \mathrm{wt} \%$ of $\mathrm{SiC}$ as additive, respectively. The chemical structure and morphology of SiC and CB samples were analyzed through Raman spectroscopy and Scanning Electron Microscopy (SEM). The dielectric properties of the RAMs were obtained in the frequency range of $12.4-18.0 \mathrm{GHz}$. The reflectivity results presented that $\mathrm{SiC} / \mathrm{CB}$ composite with $1 \mathrm{wt} \%$ of $\mathrm{CB}$ presented the best electromagnetic radiation absorption, reaching up to $-47.9 \mathrm{~dB}$ at $16.8 \mathrm{GHz}$ with a sample of $5.5 \mathrm{~mm}$ thickness.

Keywords: RAM, $\mathrm{K}_{\mathrm{u}}$-band, reflectivity, permittivity, dielectric material. 


\section{INTRODUÇÃO}

Nos últimos anos, pesquisas envolvendo Material Absorvedor de Radiação Eletromagnética (MARE), vêm sendo gradativamente desenvolvidas [1-4]. Estes materiais têm propriedades químicas e físicas [4, 5] que permitem seu comportamento como absorvedores de ondas eletromagnéticas, já que impedem a transmissão, ou o fluxo de energia, através dele em determinadas frequências. Tal impedimento ocorre através de reflexão e/ou absorção, no qual decorre da interação da onda eletromagnética (EM) tanto com a superfície do MARE quanto com o meio material. Geralmente, MAREs são formados por compósitos feitos a partir de uma matriz polimérica com aditivos de materiais com perdas dielétricas e/ou magnéticas.

Alguns materiais, tais como, Negro de Fumo (NF), nanotubo de carbono [5-7], nanoplateletes de Grafeno [8] e Carbeto de Silício (SiC) [7, 9], apresentam propriedades como MARE, pois possuem uma alta condutividade elétrica $[5,10]$ ou elevadas perdas dielétricas [11]. O negro de fumo, por exemplo, é um material que tem características propícias para MARE, pois apresenta alta condutividade elétrica [12, 13], baixa massa específica [10] e baixo custo [11]. A adição de negro de fumo aumenta a condutividade elétrica favorecendo a atenuação da onda eletromagnética [14] no compósito. Todavia, concentrações superiores a $5 \%$ em peso de NF, apresentam baixo desempenho na atenuação da onda EM $[7,12,15]$ devido ao efeito de percolação que ocorre quando as distâncias entre partículas são reduzidas significativamente, resultando em caminhos condutores no meio material. O carbeto de silício é um material cerâmico semicondutor e tem características de absorção de ondas eletromagnéticas $[16,17]$ devido às suas perdas dielétricas. Além disto, o SiC é inerte quimicamente, apresenta resistência à oxidação [2, 4, 18] e estabilidade química [2, 3, 17, 19].

O SiC e o NF são tipos de materiais amplamente investigados como MARE [3, 12, 20, 21], porém essas pesquisas são baseadas na faixa de frequência da banda $\mathrm{X}$ e, geralmente, os compósitos são feitos utilizando resina epóxi ou parafina. Desta forma, trabalhos que utilizam borracha de silicone como matriz são escassos, independente dos aditivos utilizados. Tão pouco há pesquisas sobre a influência da adição do negro de fumo ao carbeto de silício em matriz de silicone na faixa de frequência da banda $\mathrm{K}_{\mathrm{u}}(12,4-18,0 \mathrm{GHz})$, faixa de frequência de interesse em áreas de telecomunicação [7, 16], aeroespacial [5, 17, 19] e aeronáutica $[1,9,22]$.

Este trabalho apresenta a investigação da influência do carbeto de silício e do negro de fumo no estudo das propriedades dielétricas de MAREs a base de matriz de silicone, através da análise das propriedades eletromagnéticas das amostras com teor de 1 a $3 \%(\mathrm{~m} / \mathrm{m})$ de negro de fumo ao carbeto de silício. Foram feitos compósitos com três diferentes composições entre NF e SiC, que foram caracterizadas através de um analisador de rede vetorial na faixa de frequência da banda $\mathrm{K}_{\mathrm{u}}$. Com base nas propriedades intrínsicas dos materiais, os efeitos de absorção da onda foram calculados através das equações de refletividade (Reflection loss - RL) e experimentalmente validados.

\section{MATERIAIS E MÉTODOS}

\subsection{Materiais}

Para a preparação das amostras foram utilizados borracha de silicone branca da Redelease Ltda., negro de fumo da Cancarb Inc. e carbeto de silício com partículas $<44 \mu \mathrm{m}$, gentilmente cedido pela Saint Gobain.

O compósito SiC/NF foi produzido utilizando matriz de silicone variando as concentrações do aditivo de NF e SiC. Para fins de comparação, foi preparada uma amostra contendo somente $30 \%(\mathrm{~m} / \mathrm{m})$ de $\mathrm{SiC}$. A Tabela 1 mostra a identificação das amostras e a proporção dos aditivos.

Tabela 1: Identificação das amostras e proporção das misturas nos compósitos preparados. A proporção da matriz de silicone foi mantida fixa em $70 \%(\mathrm{~m} / \mathrm{m})$.

\begin{tabular}{c|c|c}
\hline Amostra & SiC $(\% \mathbf{m} / \mathbf{m})$ & NF $(\% \mathbf{m} / \mathbf{m})$ \\
\hline $\mathrm{SiC}$ & 30 & 0 \\
\hline $29 \mathrm{SiC} 1 \mathrm{NF}$ & 29 & 1 \\
\hline $28 \mathrm{SiC} 2 \mathrm{NF}$ & 28 & 2 \\
\hline $27 \mathrm{SiC} 3 \mathrm{NF}$ & 27 & 3 \\
\hline
\end{tabular}

As partículas de $\mathrm{SiC} / \mathrm{NF}$ foram dispersadas em silicone, através de agitação manual por um período de 5 minutos à temperatura ambiente até obter uma mistura uniforme. As amostras foram preparadas e 
colocadas em molde fabricado em impressora 3D, com formato retangular na dimensão $15,80 \times 7,95 \mathrm{~mm}$ (banda $\mathrm{K}_{\mathrm{u}}$ ), e, espessura aproximada de $6,0 \mathrm{~mm}$. As amostras permaneceram à temperatura ambiente por 24 horas para cura do compósito, seguido da realização de caracterização das propriedades eletromagnéticas do material.

\subsection{Caracterização dos materiais}

A caracterização morfológica e estrutural do carbeto de silício e do negro de fumo foram realizadas através de Microscopia Eletrônica de Varredura (MEV-FEG), modelo Mira3-Tescan e Espectroscopia Raman, modelo Horiba-LabRam HR Evolution usando laser com comprimento de onda de $514 \mathrm{~nm}$ e potência

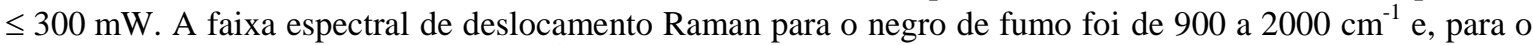
carbeto de silício foi de 600 a $1000 \mathrm{~cm}^{-1}$.

As propriedades dielétricas e a refletividade foram obtidas na faixa de frequência entre 12,4 a 18,0 GHz através de um Analisador de Rede Vetorial (Vector Network Analyzer - VNA), modelo N5235A, utilizando um kit de guia de ondas da banda $\mathrm{K}_{\mathrm{u}}$ modelo P11644A, ambos da Keysight Technologies. Na caracterização da propriedade dielétrica, a permissividade complexa $\left(\varepsilon=\varepsilon^{\prime}-j \varepsilon^{\prime \prime}\right)[4,9]$ foi obtida usando o método Nicolson Ross Weir (NRW). A razão entre as permissividades imaginária e real resulta em propriedade de atenuação da onda eletromagnética no compósito e é calculado pela equação da tangente de perda dielétrica [10]:

$$
\tan \delta=\varepsilon " / \varepsilon,
$$

A medida de refletividade foi realizada utilizando uma placa metálica refletora [12, 21], como mostrado na Figura 1.

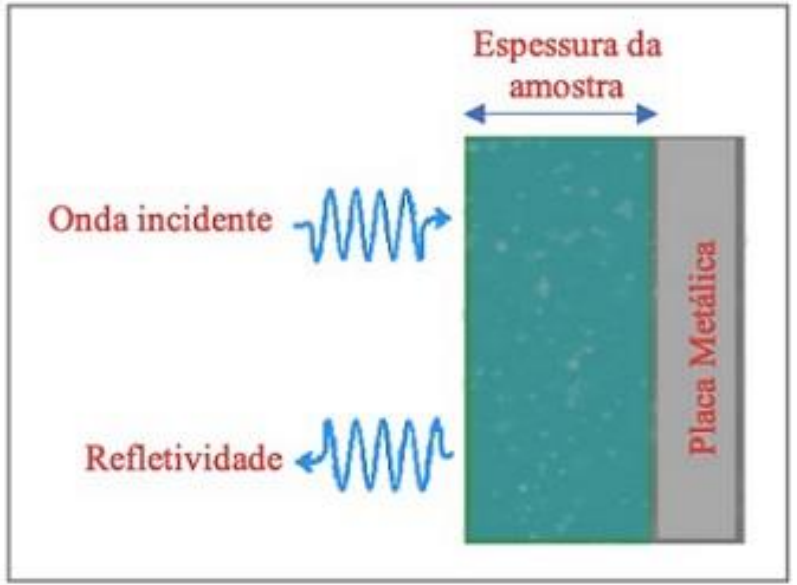

Figura 1: Esquema do mecanismo de absorção da onda eletromagnética.

O cálculo da refletividade é realizado através de [12]:

$$
R L(d B)=20 \log \left(\frac{Z_{\text {in }}-Z_{0}}{Z_{\text {in }}+Z_{0}}\right)
$$

Onde a impedância característica do ar é $Z_{0}=120 \pi$ [23] e $Z_{i n}$ é a impedância característica do material, calculada por:

$$
Z_{\text {in }}=\sqrt{\frac{\mu_{r}}{\varepsilon_{r}}} \tanh \left[j\left(\frac{2 \pi f d}{c}\right) \sqrt{\mu_{r} \varepsilon_{r}}\right]
$$

Aqui, $\mu_{r}$ é a permeabilidade relativa, $\varepsilon_{r}$ é a permissividade relativa, $c$ é a velocidade da luz no vácuo, $f$ é a frequência e $d$ é a espessura da amostra. A Tabela 2 mostra a correlação entre a porcentagem de energia absorvida e a refletividade do material [24]. 
Tabela 2: Relação entre a refletividade e o percentual de energia absorvida.

\begin{tabular}{c|c}
\hline Refletividade (dB) & \% de Energia Absorvida \\
\hline 0 & 0 \\
\hline-3 & 50 \\
\hline-10 & 90 \\
\hline-15 & 96,9 \\
\hline-20 & 99 \\
\hline-30 & 99,9 \\
\hline-40 & 99,99 \\
\hline
\end{tabular}

\section{RESULTADOS}

\subsection{Caracterização morfológica e estrutural}

O resultado da análise do $\mathrm{SiC}$ por microscopia eletrônica de varredura é mostrado na Figura 2, onde é possível verificar as características morfológicas não uniformes do material. Na Figura 2.b pode-se confirmar que as partículas de $\mathrm{SiC}$ não ultrapassam a dimensão de $40 \mu \mathrm{m}$ devido ao processo de classificação por peneiramento.
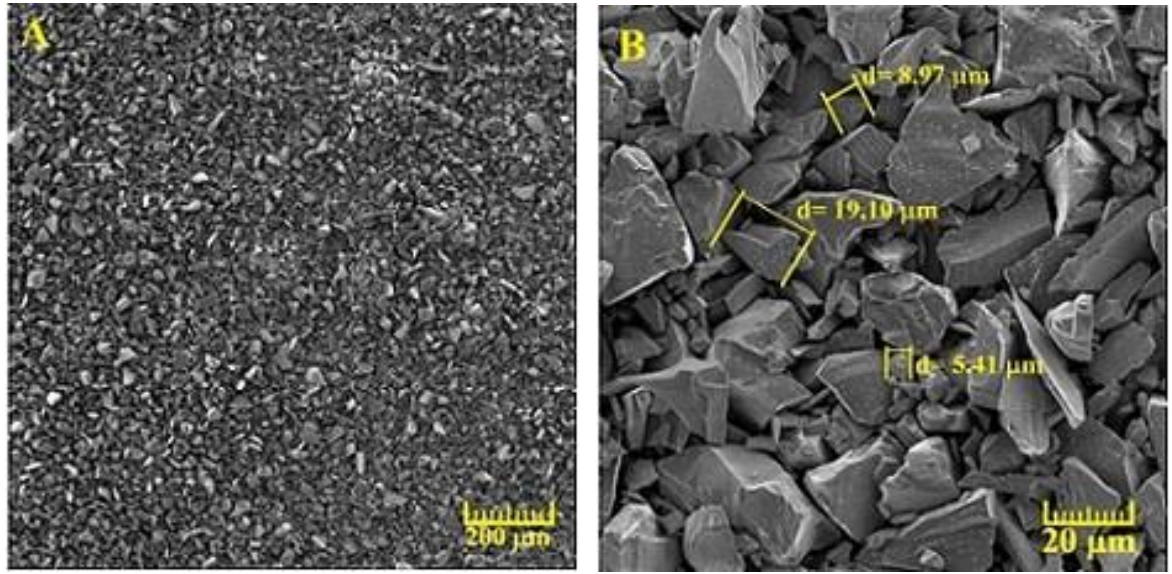

Figura 2: Morfologia das partículas do SiC com a) ampliação de 200x e b) ampliação 2000x

A Figura 3 apresenta as imagens MEV-FEG, com aspecto típico do negro de fumo. Na Figura 3.a, observamos a formação de vários grupos aglomerados em alto e baixo relevo, formado por um grande número de partículas. Isto favorece a formação de estruturas que podem beneficiar a atenuação da onda eletromagnética. A Figura 3.b mostra a geometria esférica do NF em dimensão nanométrica e, aproximadamente, $69 \mathrm{~nm}$. 

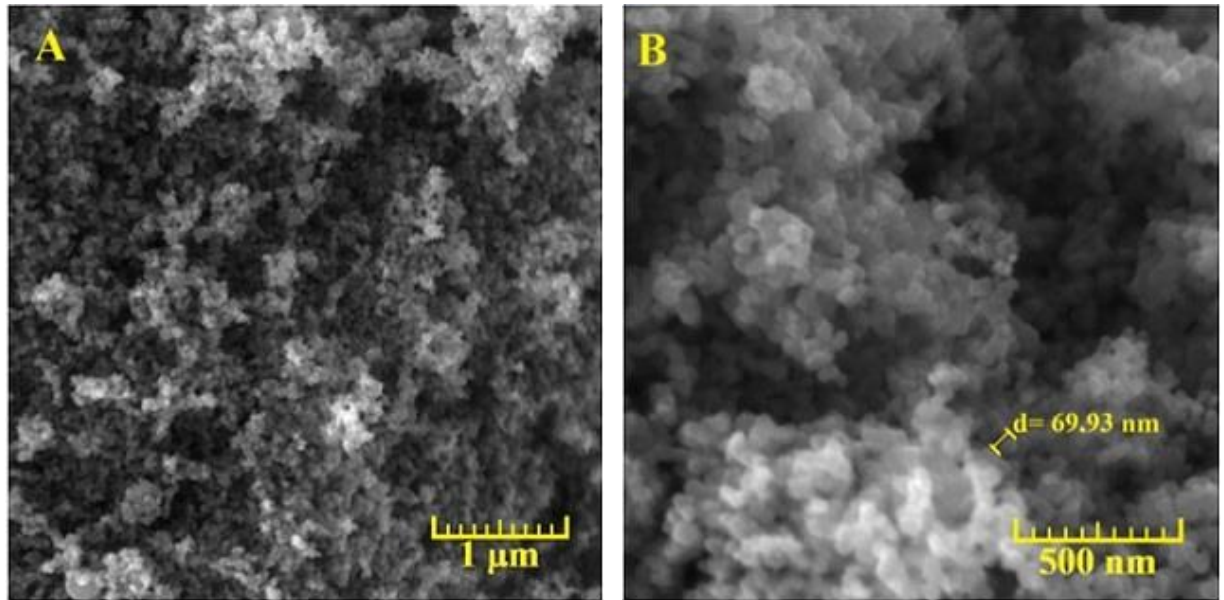

Figura 3: Morfologia das nanopartículas do NF com a) ampliação 60 kx e b) ampliação 150 kx

A espectroscopia Raman é muito utilizada na investigação de materiais carbonosos (MC). Os MC apresentam picos relevantes, para o espectro de primeira ordem, entre 1000 e $1800 \mathrm{~cm}^{-1}$, sendo as principais bandas em aproximadamente $1580 \mathrm{~cm}^{-1}$ (G) e $1350 \mathrm{~cm}^{-1}$ (D). A banda $\mathrm{G}$ exibe dupla degenerescência, porém somente a representação $\mathrm{E}_{2 \mathrm{~g}}$ é Raman ativo. A presença da banda $\mathrm{G}$ indica que o material contém carbono do tipo $\mathrm{sp}^{2}$. A banda D é associada a defeitos e não é observada para materiais grafíticos altamente orientados, ou seja, livre de defeitos [25-27]. Portanto, o espectro da Figura 4.a exibe os picos das bandas D e G para o $\mathrm{NF}$, respectivamente em 1354 e $1586 \mathrm{~cm}^{-1}$, ou seja, bandas características de materiais carbonosos [28]. O espectro da Figura 4.b apresenta os picos característicos do $\mathrm{SiC}$ em 769 e $789 \mathrm{~cm}^{-1}$ atribuídos ao modo transversal óptico (TO) e o pico em aproximadamente $973 \mathrm{~cm}^{-1}$ característico do modo longitudinal óptico (LO) [29].
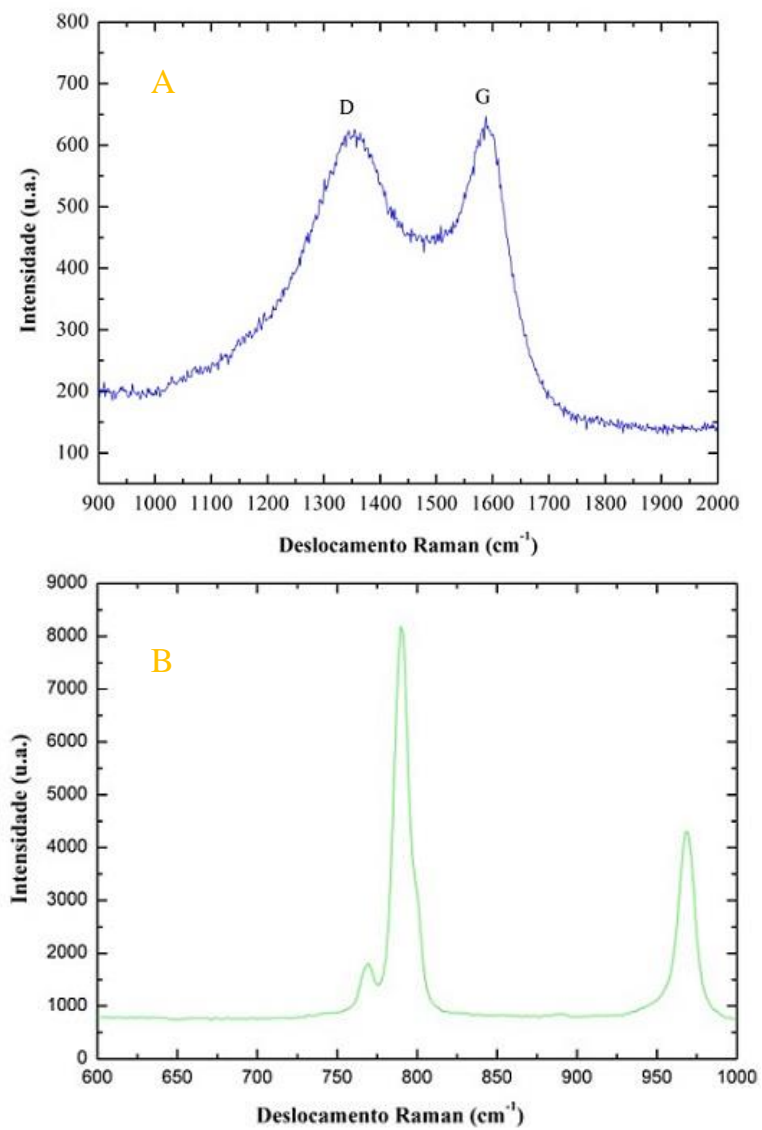

Figura 4: Espectro Raman das a) nanopartículas de NF e b) partículas de SiC. 


\subsection{Propriedades dielétricas do MARE}

As permissividades complexas das amostras são apresentadas na Figura 5. A permissividade real ( $\left.\varepsilon^{\prime}\right)$ das amostras ficaram entre 5,4 e 7,4. A permissividade imaginária ( $\varepsilon$ ') apresentou um máximo em, aproximadamente, 0,8. Foi observado que as curvas de permissividade real das amostras 27SiC3NF, 28SiC2NF e SiC diminuíram em função da frequência, enquanto a amostra 29SiC1NF apresentou uma permissividade real constante em toda a faixa de frequência da banda $K_{u}$. Devido aos efeitos de contração do processo de cura, as amostras de $\mathrm{SiC}, 29 \mathrm{SiC} 1 \mathrm{NF}, 28 \mathrm{SiC} 2 \mathrm{NF}$ e $27 \mathrm{SiC} 3 \mathrm{NF}$ apresentaram espessuras finais de $5,9 \mathrm{~mm}, 5,5 \mathrm{~mm}, 5,6 \mathrm{~mm}$ e $5,7 \mathrm{~mm}$, respectivamente.

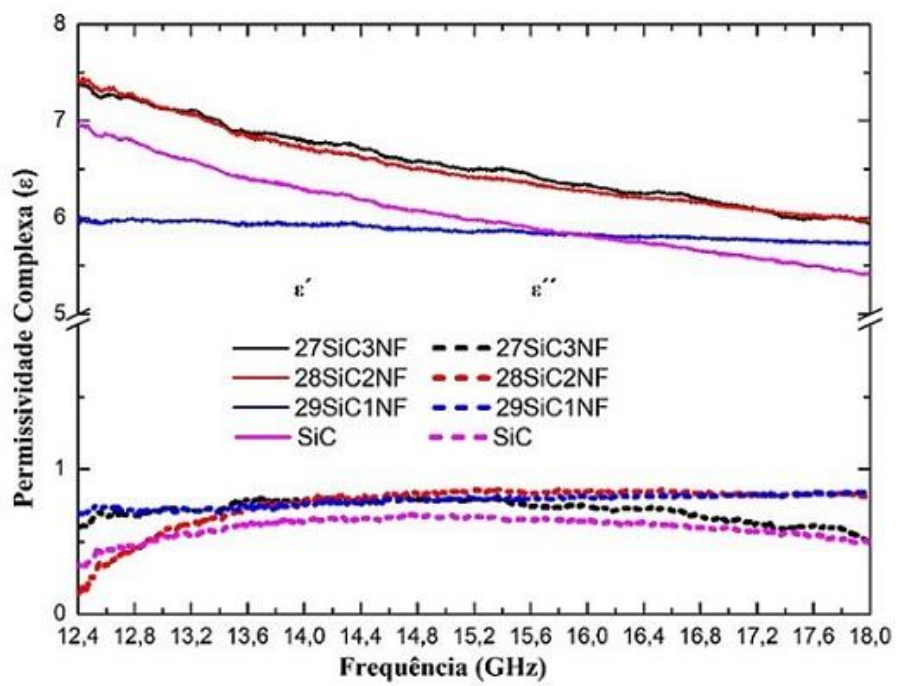

Figura 5: Permissividade complexa dos compósitos 27SiC3NF, 28SiC2NF, 29SiC1NF e SiC.

A razão entre as permissividades imaginária e real é caracterizado pela tangente de perda $(\tan \delta)$. $\mathrm{Na}$ Figura 6, observa-se que as curvas acrescem com aumento da frequência. A amostra 29SiC1NF apresentou um aumento linear em função da frequência, sendo a única que mostrou tal comportamento.

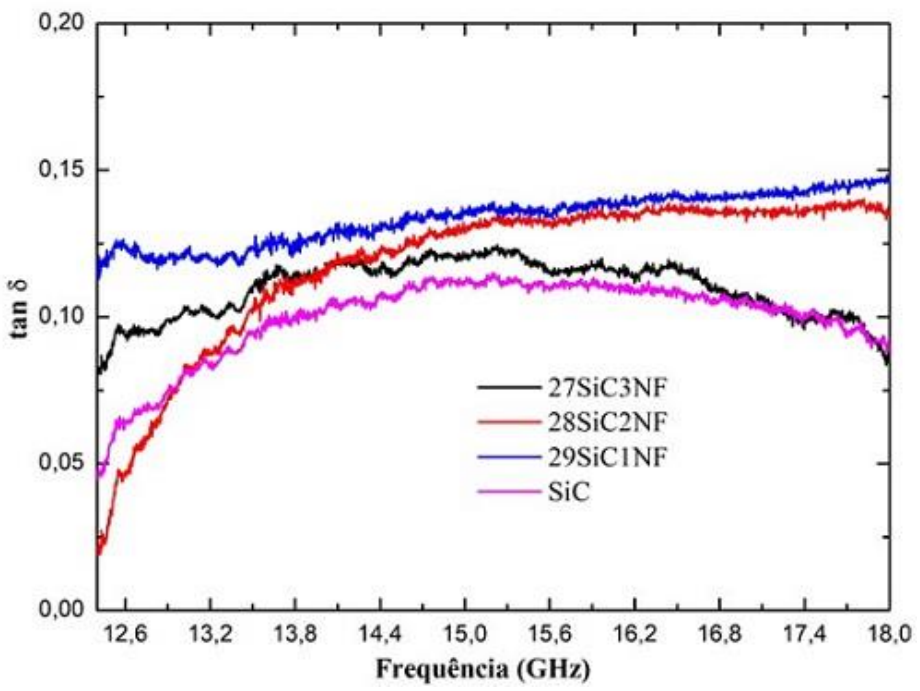

Figura 6: Curvas de perda dielétrica dos compósitos SiC/NF das amostras.

A Figura 7 apresenta os resultados experimentais e calculados de refletividade. Na análise experimental, observa-se que a amostra de $\mathrm{SiC}$ apresentou um resultado de $-35 \mathrm{~dB}$ de absorção em 15,9 $\mathrm{GHz}$, enquanto que o calculado foi de $-18,9 \mathrm{~dB}$ em $15,8 \mathrm{GHz}$. A amostra 27SiC3NF apresentou absorção de $-20,8 \mathrm{~dB}$ em 15,5 GHz na análise experimental e -25,9 dB em 15,6 dB no RL calculado, enquanto a amostra 28SiC2NF obteve resultado experimental de $-24 \mathrm{~dB}$ em $16,3 \mathrm{GHz}$ e calculado de $-25,3 \mathrm{~dB}$ em $16,5 \mathrm{GHz}$. 
Finalmente, a amostra 29SiC1NF apresentou resultado experimental de $-47,9 \mathrm{~dB}$ em 16,8 GHz e calculado de -19,2 dB em 17,1 GHz. Nota-se que os resultados experimentais e calculados de RL são próximos, sendo que a maior diferença entre eles é de $0,3 \mathrm{GHz}$ para a amostra 29SiC3NF. A largura de banda de absorção, ou seja, a faixa de frequência em que o RL<-10 dB, é de $1,71 \mathrm{GHz}, 1,82 \mathrm{GHz}, 1,91 \mathrm{GHz}$ e 1,67 GHz para as amostras 27SiC3NF, 28SiC2NF, 29SiC1NF e SiC, respectivamente.

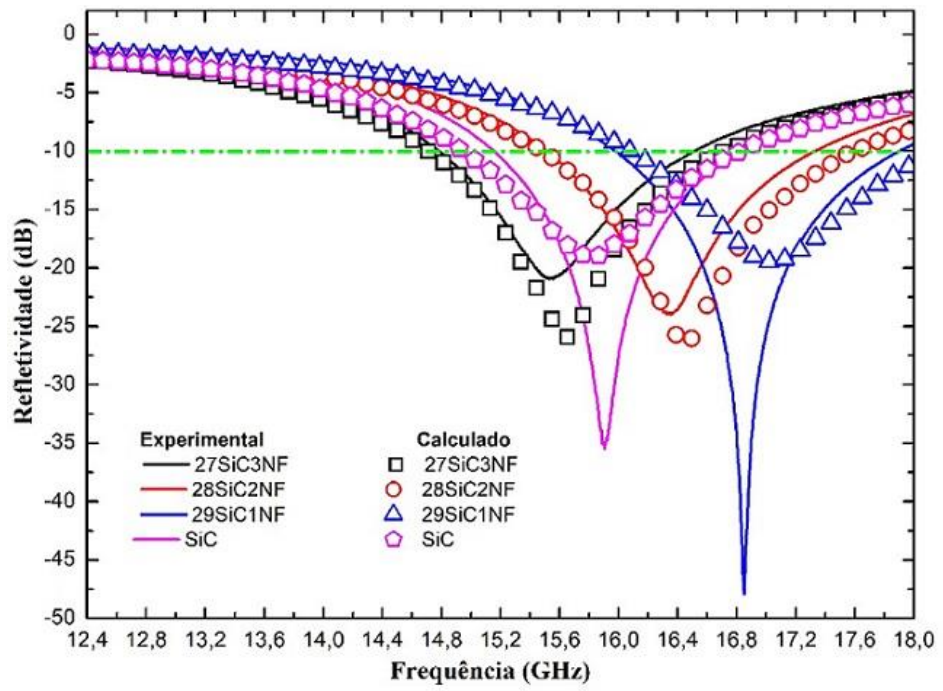

Figura 7: Curvas de refletividade do compósito SiC/NF.

\section{DISCUSSÃO}

\subsection{Características eletromagnéticas}

A adição do negro de fumo ao compósito com carbeto de silício promoveu a variação dos valores $\varepsilon$ 'e $\varepsilon$ " quando comparado ao resultado obtido para a amostra contendo somente SiC. Esse efeito pode estar relacionado com o aumento da perda dielétrica do compósito, o qual pode surgir quando há uma maior condutividade elétrica no meio material. Visto que o NF apresenta boa condutividade elétrica, sua adição pode aumentar a condutividade do compósito, promovendo uma tangente de perda maior. Desta forma, o compósito pode apresentar uma heterogeneidade, causando perdas dielétricas através da percolação elétrica [21] do aglomerado do NF.

A refletividade apresentada na Figura 7 mostra o pico de absorção do SiC entre os picos de absorção das amostras $27 \mathrm{SiC} 3 \mathrm{NF}$ e $28 \mathrm{SiC} 2 \mathrm{NF}$. Isto acontece porque a amostra de $\mathrm{SiC}$ é mais espessa que as demais, deslocando o pico para frequências mais baixas.

Experimentalmente, a adição de $1 \%(\mathrm{~m} / \mathrm{m})$ de negro de fumo no compósito apresentou o melhor resultado de atenuação da onda eletromagnética de $-47,9 \mathrm{~dB}$ de absorção em 16,8 GHz. Amostras com adição de $2 \%(\mathrm{~m} / \mathrm{m})$ e $3 \%(\mathrm{~m} / \mathrm{m})$ de negro de fumo resultam em atenuações menores: $-24,0 \mathrm{~dB}$ e $-20,8 \mathrm{~dB}$, respectivamente. Comportamentos semelhantes são mostrados por LIU et al. [12] e WAN et al. [15], onde foi observado que o aumento da concentração de negro de fumo no compósito resulta em menor absorção da onda eletromagnética, além do deslocamento do pico para frequências mais baixas. Adicionalmente, SINGH et al. [22] relatou que a dispersão de zinco ( $\mathrm{Zn})$ em apenas SiC também promoveu uma evolução na absorção de onda eletromagnética. Os dados podem ser observados na Tabela 3. 
Tabela 3: Comparação da perda por refletividade com a literatura.

\begin{tabular}{|c|c|c|c|c|c|}
\hline $\begin{array}{c}\text { Amostras } \\
(\% \mathrm{~m} / \mathrm{m})\end{array}$ & Matriz & $\begin{array}{c}\text { Espessura } \\
\text { amostra } \\
(\mathrm{mm})\end{array}$ & $\begin{array}{c}R L \\
(d B)\end{array}$ & $\begin{array}{l}\text { Frequência } \\
\qquad(\mathrm{GHz})\end{array}$ & Referência \\
\hline 29SiC1NF & \multirow{3}{*}{ Silicone } & 5,5 & $-47,9$ & 16,8 & \multirow{3}{*}{ Este trabalho } \\
\hline $28 \mathrm{SiC} 2 \mathrm{NF}$ & & 5,6 & $-24,0$ & 16,3 & \\
\hline 27SiC3NF & & 5,7 & $-20,8$ & 15,5 & \\
\hline $40 \mathrm{SiC} / 5 \mathrm{NF}$ & \multirow{3}{*}{ Parafina } & \multirow{3}{*}{2,0} & $-17,0$ & 13,0 & \multirow{3}{*}{ [12] } \\
\hline $40 \mathrm{SiC} / 10 \mathrm{NF}$ & & & $-9,5$ & 12,0 & \\
\hline $40 \mathrm{SiC} / 15 \mathrm{NF}$ & & & $-4,5$ & 9,0 & \\
\hline $2 \mathrm{NF}$ & \multirow{3}{*}{$\begin{array}{c}\text { Fibra } \\
\text { SiC/fosfato } \\
\text { de alumínio }\end{array}$} & 3,2 & $-32,0$ & \multirow{3}{*}{10,0} & \multirow{3}{*}{ [15] } \\
\hline $4 \mathrm{NF}$ & & 2,6 & $-12,5$ & & \\
\hline $6 \mathrm{NF}$ & & 2,0 & $-7,0$ & & \\
\hline $\mathrm{SiC} / 6 \mathrm{Zn}$ & \multirow{2}{*}{$\mathrm{SiC}$} & 1,7 & $-49,45$ & 15,64 & \multirow{2}{*}[22]{} \\
\hline $\mathrm{SiCZn}$ & & 2,1 & $-41,23$ & 16,16 & \\
\hline
\end{tabular}

A influência da espessura das amostras, bem como a proporção de NF no compósito SiC pode ser melhor visualizada através dos gráficos tridimensionais da Figura 8. Estes gráficos, calculados com base nas propriedades eletromagnéticas dos materiais aqui medidos, permite uma análise rápida de qual frequência e qual espessura onde cada material apresentará a melhor refletividade. A Figura 8.a mostra os resultados de refletividade da amostra de $\mathrm{SiC}$. Pode-se observar que a faixa de frequência de absorção abaixo de $-10 \mathrm{~dB}$ pode ser obtida a partir de espessuras de $5,0 \mathrm{~mm}$. Conforme há o aumento da espessura do material a refletividade se desloca para frequências mais baixas. Assim, esta amostra apresenta uma refletividade entre $13,8 \mathrm{GHz}$ e 18,0 GHz, para espessuras entre 6,5 e $5,0 \mathrm{~mm}$, respectivamente. A Figura 8.b apresenta resultados da amostra $29 \mathrm{SiC} 1 \mathrm{NF}$, aparentemente semelhantes aos resultados da Figura 8.a. Contudo, há um pequeno deslocamento em relação à faixa de espessura e um ligeiro aprimoramento da refletividade. Apesar disto, esta amostra foi a que apresentou o melhor resultado experimental de refletividade. Isto pode estar relacionado com o fato desta amostra conter uma maior proporção de $\mathrm{SiC}$ em relação ao NF. Considerando que o NF pode formar pequenos aglomerados no compósito, pode ocorrer o aumento da condutividade elétrica, por meio de percolação elétrica [21]. Isto auxilia na dispersão da energia da onda eletromagnética através de uma maior quantidade de SiC. Para a amostra 28SiC2NF, Figura 8.c, a refletividade calculada representa valores superiores às amostras de $\mathrm{SiC}$ e $29 \mathrm{SiC} 1 \mathrm{NF}$, atingindo valores abaixo de $-20 \mathrm{~dB}$, a partir de 5,2 mm de espessura. Já a amostra 27SiC3NF, Figura 8.d, apresenta uma refletividade abaixo de $-20 \mathrm{~dB}$ a partir de 5,0 mm de espessura. Apesar das amostras 28SiC2NF e 27SiC3NF apresentarem resultados teoricamente melhores, é possível que as partículas de NF, nestes compósitos, tenham se aglomerado na superfície do material até que a cura da amostra fosse completada. Isto pode ter aumentado a condutividade na superfície do compósito e, consequentemente, causado uma maior reflexão da onda incidente.
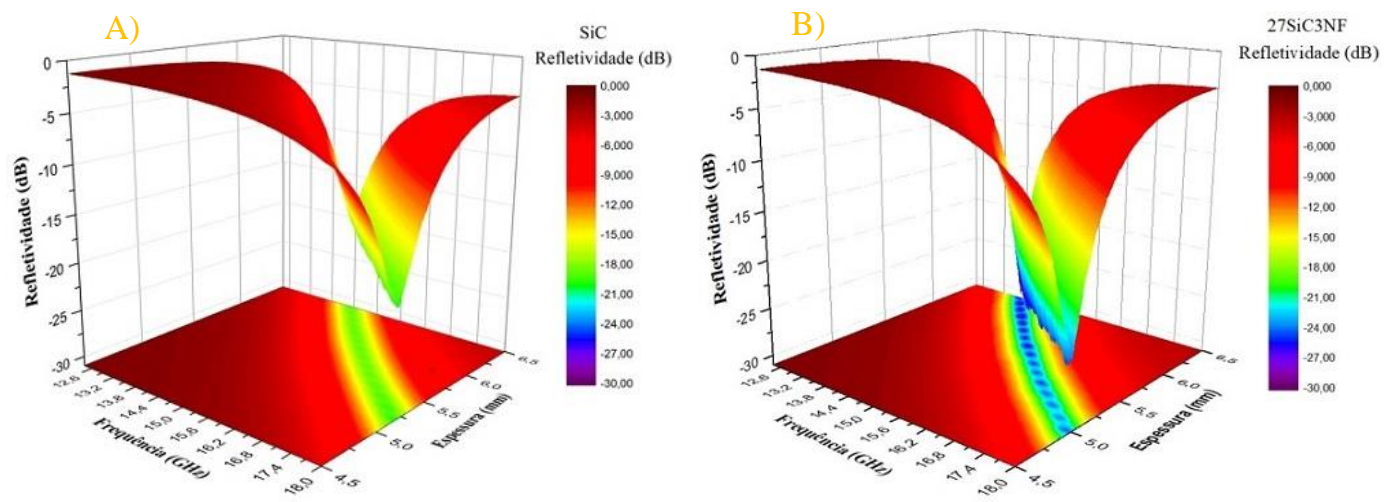

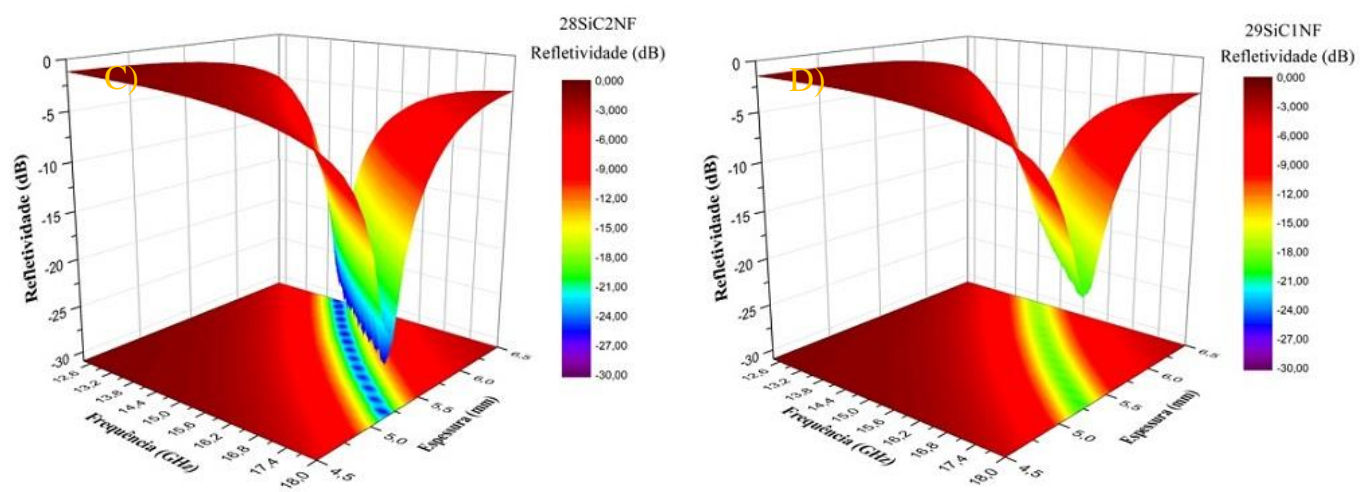

Figura 8: Curvas de refletividade calculada em função da frequência e espessura das amostras: a) SiC, b) 27SiC3NF, c) $28 \mathrm{SiC} 2 \mathrm{NF}$ e d) $29 \mathrm{SiC} 1 \mathrm{NF}$.

\section{CONCLUSÕES}

Neste trabalho, foi realizada a análise da influência da adição de negro de fumo em um compósito de silicone e carbeto de silício. A utilização do negro de fumo no MARE apresentou contribuições significativas, pois com apenas $1 \%(\mathrm{~m} / \mathrm{m})$ de adição de NF a capacidade de atenuar a radiação eletromagnética incidente foi de 99,99\% em 16,8 GHz. Ainda, a largura de banda de absorção deste material foi de 1,91 GHz. Em comparação, o SiC apresenta refletividade de $-35 \mathrm{~dB}$ em 15,9 GHz, e uma largura de banda de 1,67 GHz em $-10 \mathrm{~dB}$. Também foi discutido a razão pela qual os resultados teóricos e experimentais da amostra 29SiC1NF foram divergentes. A quantidade de NF nesta amostra possibilitou uma melhor dissipação da onda eletromagnética pelo compósito, formado majoritariamente pelo SiC. Assim, ficou demonstrado que é possivel fabricar um MARE eficiente utilizando borracha de silicone e aditivos já conhecidos na literatura, onde a vantagem da utilização do silicone é seu baixo custo e sua fácil manipulação.

\section{AGRADECIMENTOS}

Agradecimento ao Instituto Nacional de Pesquisas Espaciais (INPE), a CAPES-PNPD, CAPES-DS e ao CNPq.

Os autores, Nila e Leonardo, agradecem a Universidade Estadual de Santa Cruz (UESC).

\section{BIBLIOGRAFIA}

[1] CHEN, C.Y., PU, N.W., LIU, Y.M., et al., Microwave absorption properties of holey graphene/silicone rubber composites, Composites Part B: Engineering, 2018.

[2] ZHOU, W., YIN, R.M., LONG, L., et al., Enhanced high-temperature dielectric properties and microwave absorption of SiC nanofibers modified Si3N4 ceramics within the gigahertz range, Ceramics International, v. 44, n. 11, pp. 12301-12307, 2018.

[3] YUAN, X., CHENG, L., ZHANG, L., Electromagnetic wave absorbing properties of SiC/SiO2 composites with ordered inter-filled structure, Journal of Alloys and Compounds, 2016.

[4] WU, Q., GAO, H., ZHANG, Y., et al., Microwave absorption and mechanical properties of cross-scale SiC composites, Composites Part B: Engineering, v. 155, n. July, pp. 83-91, 2018.

[5] RAVEENDRAN, A., SEBASTIAN, M.T., RAMAN, S., "Applications of Microwave Materials: A Review", Journal of Electronic Materials, 2019.

[6] WANG, P., CHENG, L., ZHANG, L., One-dimensional carbon/SiC nanocomposites with tunable dielectric and broadband electromagnetic wave absorption properties, Carbon, 2017.

[7] GUPTA, S., TAI, N.H., "Carbon materials and their composites for electromagnetic interference shielding effectiveness in X-band”, Carbon, 2019.

[8] PLYUSHCH, A., MACUTKEVIC, J., KUZHIR, P., et al., Electromagnetic properties of graphene nanoplatelets/epoxy composites, Composites Science and Technology, v. 128, pp. 75-83, 2016.

[9] WANG, P., CHENG, L., ZHANG, Y., et al., Flexible, hydrophobic SiC ceramic nanofibers used as high frequency electromagnetic wave absorbers, Ceramics International, v. 43, n. 10, pp. 7424-7435, 2017.

[10] YANG, Z., LUO, F., GAO, L., et al., Enhanced Microwave Absorption Properties of Carbon Black/Silicone Rubber Coating by Frequency-Selective Surface, Journal of Electronic Materials, 2016. 
[11] DONG, J., ZHOU, W., DUAN, S., et al., "Mechanical, dielectric and microwave absorption properties of carbon black (CB) incorporated SiO2f/PI composites", Journal of Materials Science: Materials in Electronics, 2018.

[12] LIU, X., ZHANG, Z., WU, Y., Absorption properties of carbon black/silicon carbide microwave absorbers, Composites Part B: Engineering, v. 42, n. 2, pp. 326-329, 2011.

[13] GUPTA, K.K., ABBAS, S.M., ABHYANKAR, A.C., Carbon black/ polyurethane nanocompositecoated fabric for microwave attenuation in $\mathrm{X} \& \mathrm{Ku}$-band $(8-18 \mathrm{GHz})$ frequency range, Journal of Industrial Textiles, 2016.

[14] QIN, F., BROSSEAU, C., A review and analysis of microwave absorption in polymer composites filled with carbonaceous particles, Journal of Applied Physics, v. 111, n. 6, 2012.

[15] WAN, F., LUO, F., WANG, H., et al., Effects of carbon black (CB) and alumina oxide on the electromagnetic-and microwave-absorption properties of SiC fiber/aluminum phosphate matrix composites, Ceramics International, v. 40, n. 10, pp. 15849-15857, 2014.

[16] LI, Q., YIN, X., DUAN, W., et al., Electrical, dielectric and microwave-absorption properties of polymer derived SiC ceramics in X band, Journal of Alloys and Compounds, v. 565, pp. 66-72, 2013.

[17] YANG, H.J., YUAN, J., LI, Y., et al., Silicon carbide powders: Temperature-dependent dielectric properties and enhanced microwave absorption at gigahertz range, Solid State Communications, 2013.

[18] KUANG, J., XIAO, T., HOU, X., et al., Microwave synthesis of worm-like SiC nanowires for thin electromagnetic wave absorbing materials, Ceramics International, v. 45, n. 9, pp. 11660-11667, 2019.

[19] FRAGA, M.A., PESSOA, R.S., MASSI, M., et al., Carbeto de silício como material base para sensores mems de uso aeroespacial: Uma visão geral, Revista Materia, v. 19, n. 3, pp. 274-290, 2014.

[20] WEN, B., ZHAO, J., DUAN, Y., et al., Electromagnetic wave absorption properties of carbon powder from catalysed carbon black in X and Ku bands, Journal of Physics D: Applied Physics, 2006.

[21] MEHDIZADEH, P., JAHANGIRI, H., Effect of carbon black content on the microwave absorbing properties of CB/epoxy composites, Journal of Nanostructures, 2016.

[22] SINGH, S., SHUKLA, S., KUMAR, A., et al., Influence of Zn dispersion in SiC on electromagnetic wave absorption characteristics, Journal of Alloys and Compounds, v. 738, pp. 448-460, 2018.

[23] LIU, L., DUAN, Y., MA, L., et al., Microwave absorption properties of a wave-absorbing coating employing carbonyl-iron powder and carbon black, Applied Surface Science, 2010.

[24] BARON, C., Composites D bis J: “International Encyclopedia of Composites”. Volume 2. Von S. M. Lee. VCH Verlagsgesellschaft, Weinheim 1990. 524 S., geb. DM 450,-. ISBN 3-527-27948-2, Nachrichten aus Chemie, Technik und Laboratorium, 1991.

[25] NEIMANICH, R.J., SOLIN, S.A., First- and second-order Raman scattering from finite-size crystals of graphite, Physical Review B, v. 20, n. 2, pp. 392-401, 1979.

[26] TUINSTRA F, KOENIG JL, Raman Spectrum of Graphite, Journal of Chemical Physics, v. 53, n. 3, pp. 1126-1130, 1970.

[27] WANG, Y., ALSMEYER, D.C., MCCREERY, R.L., Raman Spectroscopy of Carbon Materials: Structural Basis of Observed Spectra, Chemistry of Materials, v. 2, n. 5, pp. 557-563, 1990.

[28] PAWLYTA, M., ROUZAUD, J.N., DUBER, S., Raman microspectroscopy characterization of carbon blacks: Spectral analysis and structural information, Carbon, 2015.

[29] BURTON, J.C., SUN, L., POPHRISTIC, M., et al., Spatial characterization of doped SiC wafers by Raman spectroscopy, Journal of Applied Physics, 1998.

\section{ORCID}

Nila Cecília de Faria Lopes Medeiros

Leonardo Iusuti de Medeiros

Aparecida Teixeira de Souza

Guilherme Frederico Bernardo Lenz e Silva

Alan Fernando Ney Boss

Gisele Aparecida Amaral-Labat

Maurício Ribeiro Baldan https://orcid.org/0000-0001-7266-3936

https://orcid.org/0000-0002-1297-9063

https://orcid.org/0000-0002-5893-2885

https://orcid.org/0000-0003-1184-5271

https://orcid.org/0000-0002-4600-5443

https://orcid.org/0000-0003-3745-6119

https://orcid.org/0000-0001-7605-1064 